\title{
Salt and geographical mortality of gastric cancer and stroke in Japan
}

\author{
SUMINORI KONO, MASATO IKEDA, AND MICHIHARU OGATA \\ From the Department of Public Health, Faculty of Medicine, Kyushu University, Fukuoka, Japan
}

SUMMARY Age- and sex-adjusted mortality rates of gastric cancer and stroke in the years 1974-6 for 46 prefectures and 12 regions in Japan were related to regional differences of average per capita daily intake of salt during the period 1966-70 to test Joossen's hypothesis that salt is a common cause of both gastric cancer and stroke. While mortality rates of stroke were strongly correlated with salt intake in 12 regions $(r=0 \cdot 85, p<0 \cdot 001)$, mortality rates of gastric cancer showed hardly any correlation with salt intake $(r=0 \cdot 02)$. There was no strong correlation between the two diseases in perfectural or regional mortality rates $(r=0 \cdot 20)$. It was concluded that geographical mortality of gastric cancer and stroke in Japan did not support Joossens's hypothesis, and other evidence against the salt hypothesis has also been referred to.

Although the mortality rates of both gastric cancer and stroke have declined in most industrialised countries for the last decades, these two diseases are still the leading causes of death in Japan. Gastric cancer and stroke accounted for over $30 \%$ of all deaths in Japan in $1978 .{ }^{1}$ The aetiology of gastric cancer is essentially still not known despite discussion of various possible risk factors or protective factors in publications. ${ }^{23}$ Raised blood pressure is known to be a principal risk factor of stroke, but the cause of hypertension still remains in dispute.

Observing the similarity of the world-wide geographical distribution for the two diseases and of the secular trends, Joossens proposed that gastric cancer and stroke might be aetiologically linked and that excessive salt intake might be a common cause..$^{4-6}$ In fact, salt has so far been the most probable candidate for the cause of raised blood pressure despite the controversy over it $^{4}$ ( $R$ O Cummins, unpublished observations). Although evidence is limited, salt has also been claimed as a predisposing factor for gastric cancer in some epidemiological studies ${ }^{7-9}$

In view of the massive impact of stomach cancer and stroke on public health in Japan, Joossens's hypothesis could have a potential value in preventing the two diseases. This paper tests Joossens's hypothesis more directly by comparing geographical distribution of these two diseases with salt intake in Japan.

\section{Materials and methods}

Numbers of deaths from gastric cancer (8th ICD 151) and stroke (8th ICD 430-438), by sex, five-year age group, and prefecture during 1974-6 were used with the 1975 census population to calculate average annual death rates. ${ }^{10}{ }^{11}$ Since data of salt intake were not available at the level of prefecture but of region (fig 1), prefectural mortality data were also amalgamated for each region. The age range 45-74 years was included here because deaths from gastric cancer and stroke are few below 45 years of age, and

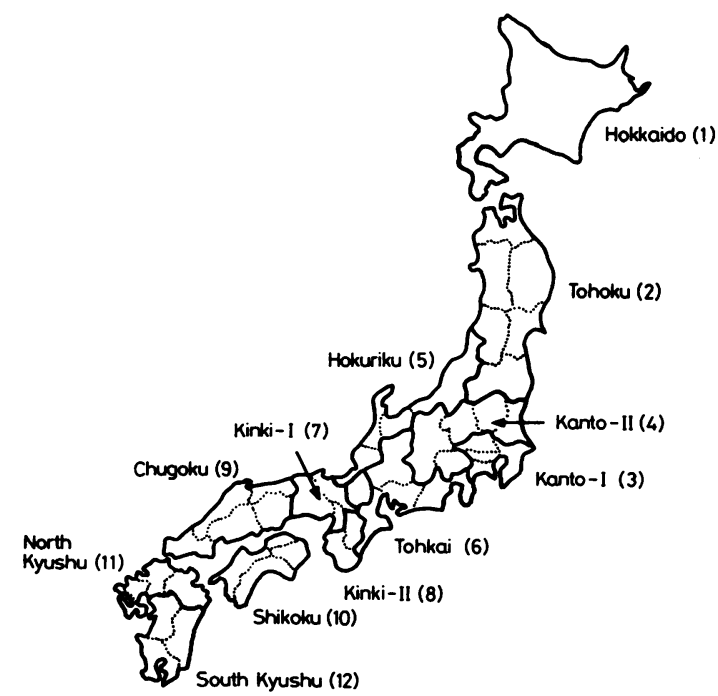

Fig 1 Japan: Regions and prefectures are delineated by straight and dotted lines, respectively. Okinawa Prefecture is excluded. Number in parentheses is used for showing corresponding region in other figures. 
death certification in the elderly is known to be inaccurate. Average annual age-adjusted death rates were calculated for 46 prefectures and 12 regions by direct method with the 1975 census population of Japan as the standard. Since male and female age-adjusted death rates for 46 prefectures were strongly correlated for both gastric cancer and stroke $(r=0.86$ and $r=0.92$, respectively), age- and sex-adjusted death rates are presented.

Date of per capita salt intake for 12 regions in five years (1966-70) were obtained from the calculation by Kikuchi et $\mathrm{al}^{12}$ based on the results of the National Nutrition Surveys, and regional averages for this period were calculated. In the National Nutrition Survey, annually carried out by the Ministry of Health and Welfare, roughly 15000 households in about 340 areas randomly selected throughout the country are asked to record the type and amount of foods consumed over five days, and the average per capita daily amount of intake is tabulated for each group of foods. ${ }^{13}$

\section{Results}

Prefectures in the regions with higher salt intake generally had higher mortality rates of stroke, but this was not so with gastric cancer (fig 2). In South Kyushu, where prefectures with the lowest mortality rates of gastric cancer were concentrated, salt intake was not low but was about the national average. Also, in prefectures in the two regions with the lowest salt intake, Kinki-I and Kinki-II, mortality rates of gastric cancer were fairly high.

The relationships of salt intake with stroke and gastric cancer are shown more clearly in figs 3 and 4 . Although mortality rates of stroke in 12 regions were highly correlated with salt intake $(r=0.85$, $\mathrm{p}<0.001)$, the rates of gastric cancer showed no correlation with salt intake $(r=0 \cdot 02)$. As expected from the findings above, the correlation between mortality rates of gastric cancer and stroke was weak in the comparisons of both 46 prefectures and 12 regions with coefficients of 0.20 in both cases.

\section{Discussion}

Although the current study has the advantage of using uniform data sources with a single country, there are admittedly several problems to be noted in interpreting the results. Because there is a fairly wide variation of mortality rates of gastric cancer and stroke between prefectures within certain regions

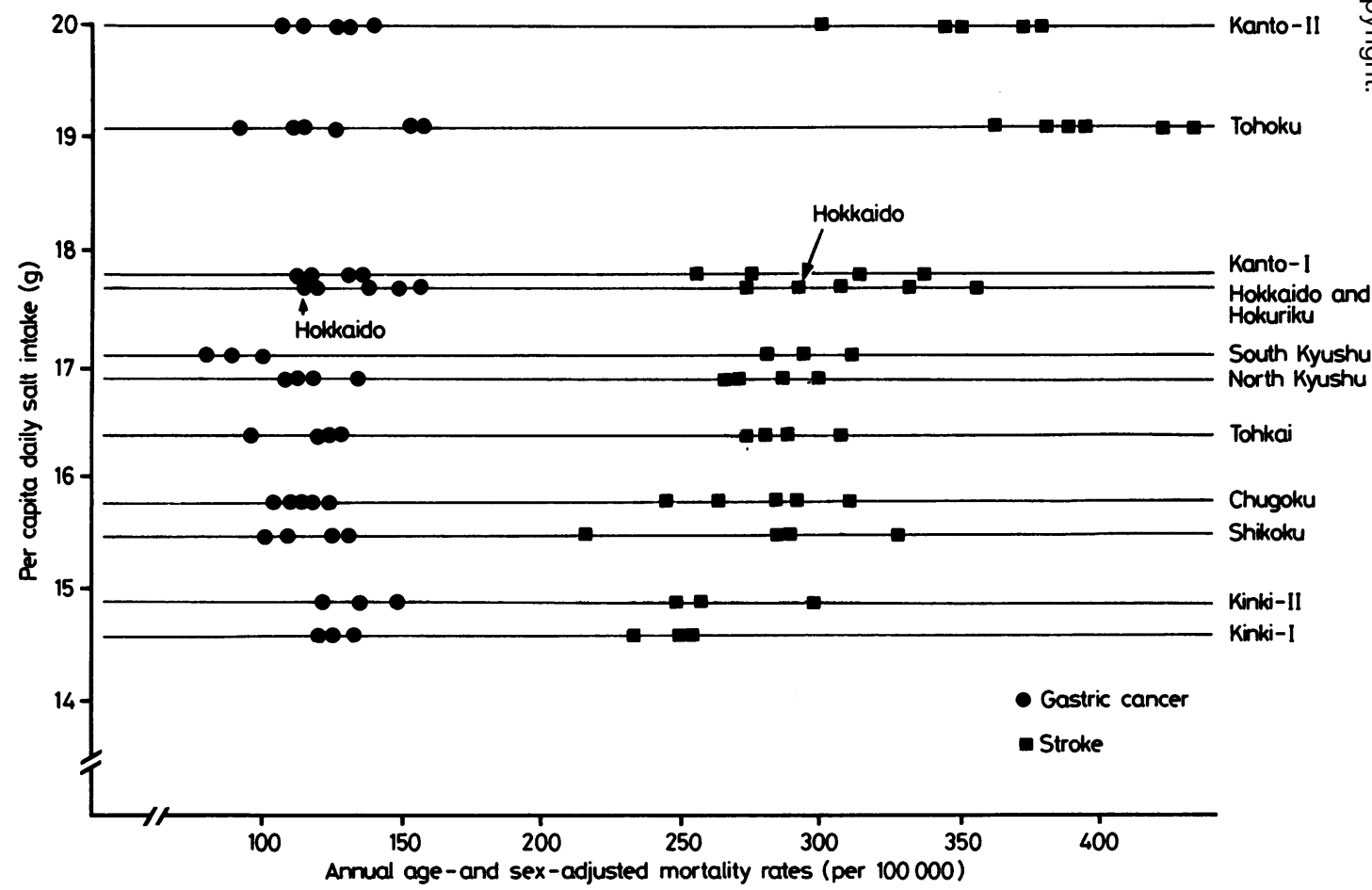

Fig 2 Distribution of annual age- and sex-adjusted mortality rates of gastric cancer and stroke in 46 prefectures, $1974-6$ against average salt intake in 12 regions, 1966-70. 


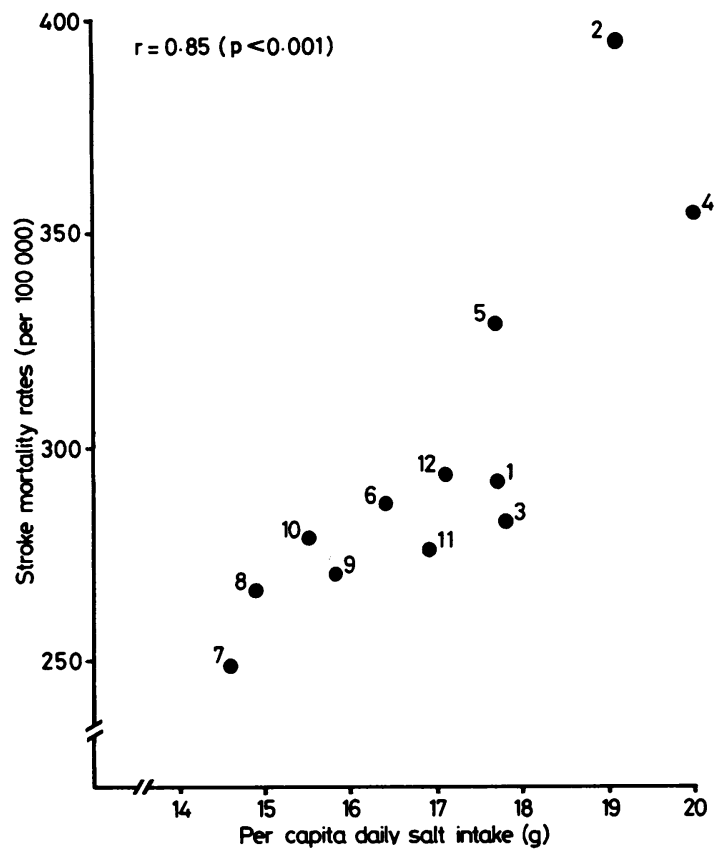

Fig 3 Annual age-and sex-adjusted mortality rates of stroke and average salt intake in 12 regions. (Number attached to dot stands for region in fig 1.)

particularly gastric cancer in Tohoku and stroke in Shikoku, the amalgamation of prefectures might weaken the relationship of salt intake with the two diseases. It is unlikely, however, that the lack of correlation between salt intake and mortality rates of gastric cancer is due to this amalgamation, because stroke mortality showed a fairly strong correlation with salt intake. Furthermore, the strong regional correlation between salt intake and mortality rates of stroke observed here is consistent with the previous studies in different geographical units in Japan-that is, 11 farm districts of Japan or administrative areas within a single prefecture..$^{14} 15$ Therefore, some confidence is given to the validity of the present analysis.

In the current analysis no other factors than salt intake were taken into account. Social class is well known to be inversely related to both gastric cancer and stroke. ${ }^{6}$ Nevertheless, differing distribution of the social classes in the regions, if any, would not explain the lack of correlation between salt intake and only one of the two diseases with similar relationship to social classes. Although high intake of milk or vitamin $\mathrm{C}$ among others has been reported as a protecting factor in the development of gastric cancer, ${ }^{3}$ South Kyushu with the lowest mortality rates of gastric cancer and the medium level of salt intake

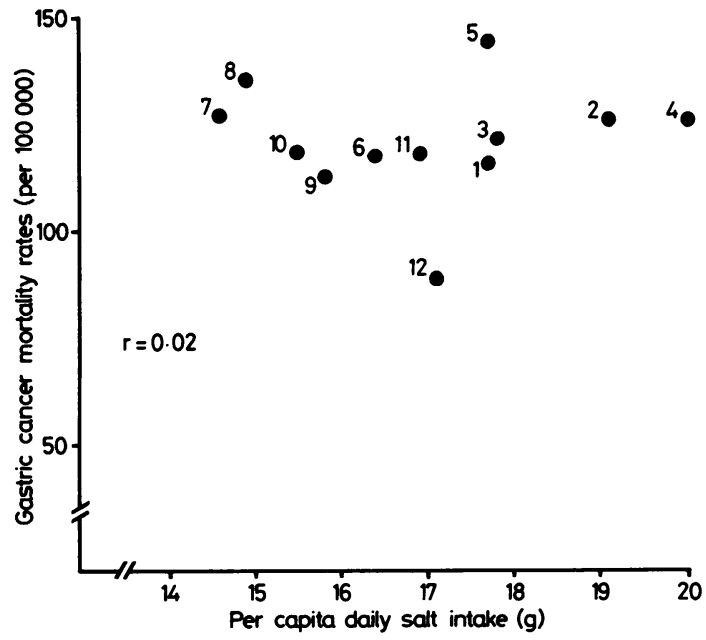

Fig 4 Annual age- and sex-adjusted mortality rates of gastric cancer and average salt intake in 12 regions. (Number attached to dot stands for region in fig 1.)

has a generally lower intake of milk or vitamin $\mathrm{C}$ than the national average. ${ }^{13}$ Since the geographical pattern of gastric cancer mortality in Japan, unlike that of stroke mortality, seems unable to be explained by regional difference in salt intake, salt is not considered to play as important a part in the causation of gastric cancer as in stroke.

There is also some evidence against Joossens's hypothesis regarding secular trends of gastric cancer and stroke. Mortality rates of the two diseases have linearly declined in Japan since the early $1960 s,{ }^{5}$ but dietary salt consumption in Japan is not considered to have decreased over the past decades, which is contrary to the general belief that salt consumption has been reduced due to the introduction of refrigeration techniques. According to the data from Sasaki and Kikuchi' ${ }^{16}$ based on salt sales during 1956-74, per capita salt consumption in Japan has shown an upward trend since around the mid-1960s owing to the increased use in food manufacturing, although the consumption of table salt gradually decreased over the period. A similar argument has also been provided for salt consumption in the United Kingdom ( $\mathrm{R}$ O Cummins, unpublished observations).

Interestingly, a recent study of multiple causes of death in England and Wales has failed to find a greater concordance on death certificates between gastric cancer and stroke than between lung cancer or pancreatic cancer and stroke. ${ }^{17}$

Therefore, the evidence so far accumulated does not necessarily support Joossens's hypothesis that salt intake is a common aetiological agent of both 
gastric cancer and stroke, although the issues might need further classification.

Reprints from Dr S Kono, Department of Public Health, Faculty of Medicine, Kyushu University, Fukouka 812, Japan.

\section{References}

${ }^{1}$ Ministry of Health and Welfare. Vital statistics 1978, Japan. Vol. 2. Tokyo: Health and Welfare Statistics Association, 1979.

${ }^{2}$ Haenszel W, Correa P. Developments in the epidemiology of stomach cancer over the past decade. Cancer Res 1975; 35: 3452-9.

${ }^{3}$ Piper DW. Stomach cancer. UICC technical report series. Vol 34. Geneva: International Union Against Cancer, 1978: 5-26.

${ }^{4}$ Joossens JV. Dietary salt restriction. The case in favour. In: Robertson JIS, Pickering GW, Caldwell ADS, eds. The therapeutics of hypertension. London: Academic Press and Royal Society of Medicine, 1980: 243-50.

${ }^{5}$ Joossens JV. Stroke, stomach cancer and salt. A possible clue to the prevention of hypertension. In: Kesteloot $\mathbf{H}$, Joossens JV, eds. Epidemiology of arterial blood pressure. The Hague: Martinus Nijhoff Publishers, 1980: 489-508.

${ }^{6}$ Joossens JV, Geboers J. Nutrition and gastric cancer. Nutr Cancer 1981; 2, 250-61.

${ }^{7}$ Sato, T, Fukuyama T, Suzuki T, et al. Studies of the causation of gastric cancer. 2 . The relationship between gastric mortality rate and salted food intake in several places in Japan, Bull Inst Public Health 1959; 8: 187-98.
${ }^{8}$ Hirayama T. Epidemiology of stomach cancer. In: Japan Cancer Association, ed. Gann monograph on cancer research No 11. Tokyo: Japan Scientific Society Press, 1971: 3-19.

${ }^{9}$ Haenszel W, Kurihara M, Segi M, Lee RKC. Stomach cancer among Japanese in Hawaii. J Natl Cancer Inst 1972; 49: 969-88.

${ }^{10}$ Ministry of Health and Welfare. Vital statistics in Japan. Numbers of deaths by cause, sex, age and prefecture, 1974-76 Tokyo: Health and Welfare Statistics Association. (A series of microfilms.)

${ }^{11}$ Bureau of Statistics, Office of the Prime Minister. 1975 population census of Japan. Vol 2. Tokyo: Health and Welfare Statistics Association, 1977.

${ }^{12}$ Kikuchi R, Togashi M, Konno K. Salt intake and its constitution based on the national nutrition surveys. The proceedings of 23rd conference of the Japan Dietetic Society, Nagoya, 1976. Tokyo: The Japan Dietetic Society, 1976: 218-9.

${ }^{13}$ Ministry of Health and Welfare. The present state of national nutrition. The reports of National Nutrition Survey, 1966-70. Tokyo: Ministry of Health and Welfare, 1968, 1969, 1970, and 1973.

${ }^{14}$ Fujisaku S, Ito T, Kamoi M, Hiraide H. Age-adjusted death rates for cerebral apoplexy by cities, towns and villages in Miyagi Prefecture in 1955-8 Tohoku Igaku Zasshi 1960; 61: 628-34.

${ }^{15}$ Sasaki N. The relationship of salt intake to hypertension in the Japanese. Geriatrics 1964; 19: 735-44.

${ }^{16}$ Sasaki N. Kikuchi R. Salt and nutrition. Tokyo: Dai-ichi Shuppan Co, 1980: 86.

${ }^{17}$ Whelton PK. Goldblatt P. An investigation of the relationship between stomach cancer and cerebrovascular disease. Evidence for and against the salt hypothesis. Am J Epidemiol 1982; 115: 418-27. 